\title{
OPTICAL MONITORING OF ACTIVITY FROM MANY AREAS OF THE IN VITRO AND IN VIVO SALAMANDER OLFACTORY BULB: A NEW METHOD FOR STUDYING FUNCTIONAL ORGANIZATION IN THE VERTEBRATE CENTRAL NERVOUS SYSTEM $^{1}$
}

\author{
HARRY S. ORBACH ${ }^{2}$ AND LAWRENCE B. COHEN
}

Department of Physiology, Yale University School of Medicine, New Haven, Connecticut 06510

Received March 7, 1983; Revised May 9, 1983; Accepted May 9, 1983

\begin{abstract}
We have investigated the use of voltage-sensitive dyes to monitor neuronal activity in the intact salamander olfactory bulb. After a 10- to 20-min staining period, a magnified image of an in vitro or an in vivo preparation was formed on a 124-element photodiode array. The array was used to simultaneously record absorption or fluorescence changes from 124 adjacent areas of the bulb. At the magnifications used, each detector received light from 100 to 1000 neurons. Relatively large absorption and fluorescence signals were found in response to olfactory nerve stimulation; all of the results presented were from single trials. Because of the large signal size, measurements on in vivo preparations using epi-illumination also had good signal-to-noise ratios. There were significant differences in signal time course between adjacent detectors which suggested a spatial resolution on the order of $200 \mu \mathrm{m}$. Tentative assignments of the cellular origins of some signals could be made from the results of paired volley experiments. The results suggest that optical monitoring of membrane potential could provide a useful method for studying neuronal organization in the intact vertebrate central nervous system.
\end{abstract}

Our understanding of brain function might be enhanced by a more detailed monitoring of brain activity. A useful method would (1) have the time resolution to monitor electrical activity; (2) have a fine enough spatial resolution $(\sim 100 \mu \mathrm{m})$ to resolve functional units, e.g., cortical columns, or gross features of sensory and motor maps; and (3) be able to monitor activity at many sites simultaneously. These goals might be achieved by using voltage-sensitive dyes to monitor changes in membrane potential. Changes in absorption or fluorescence of these dyes, when bound to membranes, are proportional to the change in voltage across that membrane. For certain

\footnotetext{
${ }^{1}$ We are grateful to John Kauer for suggesting the in vitro salamander bulb, demonstrating its viability and electrophysiology, and for helpful conversations. A. Grinvald and L. Anglister very kindly informed us of experiments similar to ours which they had carried out on goldfish optic tectum. We thank $D$. Bowling and M. Nowycky for helpful suggestions during the course of the experiments and Joshua Freedman, Amiram Grinvald, John Kauer, Rill Ross, Brian Salaherg, and Gordon Shepherd for comments about the manuscript. This work was supported by Grant NSO8437 from the National Institute of Neurological and Communicative Disorders and Stroke.

${ }^{2}$ To whom correspondence should be addressed.
}

dyes these optical signals follow changes in membrane potential with a delay of less than $0.01 \mathrm{msec}$ (for a review see Cohen and Salzberg, 1978).

Potentiometric dyes can be used to monitor activity in many areas simultaneously by projecting an image of the preparation onto an array of photodetectors. Monitoring the photodetector outputs will then result in a simultaneous measure of the average membrane potential of the membranes whose image falls on each detector. This technique has already been used at the subcellular level to investigate neuron processes in invertebrate ganglia and in tissue culture (Grinvald et al., 1981a; Krauthamer and Ross, 1982, 1983; Ross and Krauthamer, 1983); it has been used at the cellular level to simultaneously monitor the activity of individual neurons in invertebrate ganglia (Salzberg et al., 1977; Grinvald et al., 1981b); and it has been used at the multicellular level in hippocampal slices to measure the spread of activity resulting from point stimuli (Grinvald et al., 1982a). At the magnifications used in the experiments reported here, each photodetector received light from a square area of salamander olfactory bulb that was between 45 and $133 \mu \mathrm{m}$ on a side. From counts of neurons in the olfactory bulb (J. S. 
Kauer, unpublished results), we estimate that light passing through between 100 and 1000 bulb neurons was imaged on each detector. Therefore, we would not expect to detect the signals of individual neurons; any signals are likely to represent summed responses from simultaneously active neurons. In this respect, the measurements reported here are similar to those obtained from hippocampal slices (Grinvald et al., 1982a).

Potentiometric dye methods may have advantages over other techniques that have been used to study neuronal organization. These optical measurements have excellent time resolution and are made in real time on living preparations, unlike 2-deoxyglucose (Sokoloff, 1977) or cytochrome oxidase (Wong-Riley, 1979) measures of activity. Measurement of the NAD/NADH ratio using fluorescence provides another method for monitoring activity-dependent changes in metabolism. However, the time resolution is in the range of seconds to minutes (Aubert et al., 1964; Mayevsky et al., 1982).

Optical measurements of potential can have good spatial resolution; in certain preparations, resolution at the cellular or subcellular level is obtained. Thus, in principle, an optical method may provide as good a resolution as 2-deoxyglucose or cytochrome oxidase methods and better resolution than that for presently available technology for positron emission tomography, nuclear magnetic resonance, computer-assisted tomography, electroencephalography, and magnetoencephalography $(1,000$ to $10,000 \mu \mathrm{m})$ (Raichle, 1979; Wood, 1981; Kaufman and Williamson, 1982). However, in practice, in preparations with substantial light-scattering, like vertebrate brain, the resolution of an optical method will be degraded from that obtained in transparent preparations.

The goal of this investigation was to test the use of optical techniques on an intact part of a vertebrate brain. We began with an in vitro salamander bulb preparation suggested to us by John Kauer who demonstrated the viability of the preparation and showed that the field potentials in the in vitro bulb were similar to those in the in vivo preparation (J. S. Kauer, personal communication).

The salamander olfactory bulb offers several advantages for an initial attempt to measure optical signals from an intact brain area. First, the availability of both an in vitro and in vivo preparation (Mori and Shepherd, 1979; Waldow et al., 1981) enabled us to begin with a situation that avoids noise from circulatory movements and later to evaluate the severity of this interference. Second, suprathreshold stimulation of the olfactory nerve results in very large field potentials (up to $30 \mathrm{mV}$ ) in the bulb, suggesting that a relatively large fraction of the neurons is simultaneously activated. A large percentage of active cells increases the probability of being able to detect optical signals. Third, the salamander bulb was transparent enough to allow absorption measurements using transmitted light. With the dyes and apparatus presently in use, absorption signals were better than fluorescence or birefringence in terms of signal-to-noise ratio and photodynamic damage in experiments on invertebrate ganglia (Salzberg et al., 1977; Boyle and Cohen, 1980; Grinvald et al., 1981a). In addition to absorption, we wanted to test the use of epi (incident light)-illumination for both fluorescence and absorption since this kind of illumination would be needed if the technique were to be used with more opaque, mammalian preparations.

Because the cell layers in the salamander olfactory bulb are oriented perpendicular to the surface (Herrick, 1965), different diodes will receive light from different cell types and, therefore, some spatial segregation of signals may be anticipated.

A preliminary account of these experiments has been presented (Orbach and Cohen, 1982).

\section{Materials and Methods}

Preparation. Water phase tiger salamanders, Ambystoma tigrinum (15 to $40 \mathrm{gm}$ ), were anesthetized for approximately $20 \mathrm{~min}$ in a solution of Finquel (tricaine methanesulfonate) (Ayerst Laboratories, New York, NY) until they no longer responded to a foot pinch $(0.15$ to $0.5 \mathrm{gm} /$ liter for in vivo; 0.5 to $2.0 \mathrm{gm} /$ liter for in vitro). The animals were wrapped in paper towels saturated with the anesthetic solution and placed in a head holder (Kauer, 1974). The skull was opened dorsally and the dura and arachnoid membranes were removed. For the in vitro experiments, the olfactory nerves were cut as distally as possible within the cranial cavity; the caudal cut varied from midway in the telencephalic hemispheres to the rear of the tectum. The preparation was placed in air-equilibrated Ringer's $(90 \mathrm{mM} \mathrm{NaCl}, 2.5 \mathrm{mM} \mathrm{KCl}, 2$ $\mathrm{mM} \mathrm{CaCl}, 10 \mathrm{~mm} \mathrm{NaHCO} 3,0.5 \mathrm{mM} \mathrm{NaH}_{2} \mathrm{PO}_{4}, 1.0 \mathrm{~mm}$ $\mathrm{MgCl}_{2}, 10 \mathrm{mM}$ dextrose) (Sakin and Boulpaep, 1981) and pinned to the Sylgard bottom of a chamber, which was then attached to the stage of a Leitz Ortholux II microscope mounted on a vibration isolation table. A similar anesthetization and operating procedure was used for the in vivo experiments. The whole animal was then put on the microscope stage with its cranium opened and bulb in view.

Optical methods. The apparatus for optical recording has been described previously (Grinvald et al., 1981b). The four different configurations used in the experiments reported here are shown diagrammatically in Figure 1. All four mcasurements were made using the Leitz microscope.

Figure $1 A$ shows the configuration for measurements of absorption of transmitted light using brightfield illumination. The light was made quasimonochromatic by an interference filter (Filter 1) placed between the light source and the preparation. The image of the preparation was focused by the microscope objective onto the photodetector array. The measurement of fluorescence using brightfield illumination is illustrated in Figure $1 B$. Here a long-wavelength pass filter (Filter 2) was used to block the incident wavelengths but pass the emitted wavelengths. For preparations too opaque for brightfield illumination, epi-illumination is necessary. In epi-illumination (Fig. 1, $C$ and $D$ ), the incident light was reflected down onto the preparation through the objective using a mirror in the objective carrier. Figure $1 C$ shows the configuration for measurement of changes in absorption (by measuring changes in reflected light) using a quartersilvered mirror. For measurements of fluorescence using epi-illumination (Fig. $1 D$ ) a dichroic mirror is superior 
A. TRANSMISSION ABSORPTION
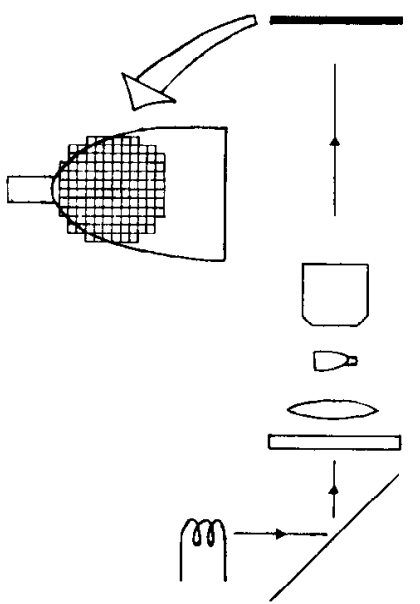

B. TRANSMISSION FLUORESCENCE

$$
\begin{aligned}
& \text { C. EPI- } \\
& \text { ABSORPTION }
\end{aligned}
$$

D. EPIFLUORESCENCE
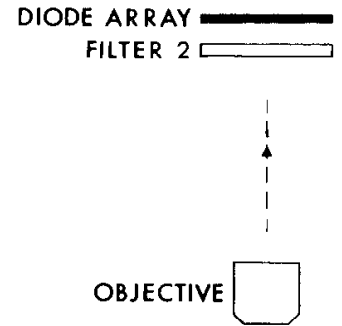

PREPARATION DO

CONDENSER $\longrightarrow$ FILTER 1

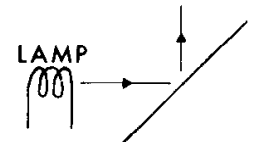

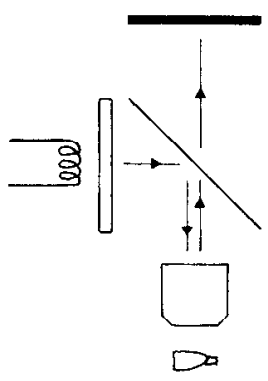

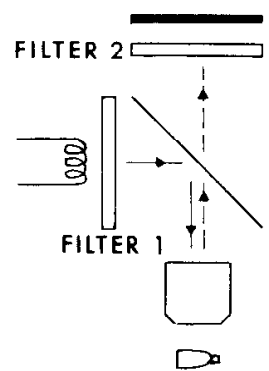

Figure 1. Schematic diagram of the four optical configurations used in these experiments. In $A$ and $C$, absorption was measured; in $B$ and $D$, fluorescence was measured. In $A$ and $B$, ordinary brightfield illumination was used; in $C$ and $D$, epi (incident light)illumination was used. A quarter-silvered mirror was used in $C$; the dichroic mirror in $D$ reflected short wavelengths (excitation) and transmitted long wavelengths (emission). The mirror (Omega Optical, Brattleboro, VT) that was used for the experiments illustrated in Figures 4 and 6 had $50 \%$ reflectance wavelength of $570 \mathrm{~nm}$. The diode array was a 144-element array, each element $1.4 \times 1.4 \mathrm{~mm}$ (purchased from Centronic, Ltd., Craydon, U. K.), 124 of the elements were used; 20 elements in the corners were not connected. Additional details of the apparatus were described previously (Grinvald et al., 1981b).

to a quarter-silvered mirror. A dichroic mirror can reflect almost all of the light at the exciting wavelengths and transmit almost all of the light at the emission wavelengths, in contrast to the quarter-silvered mirror where $75 \%$ of the incident and $25 \%$ of the fluorescent light is lost. Some measurements of fluorescence using epi-illumination were made without an incident light filter (Filter 1) because the wavelength dependence of the reflectance of the dichroic mirror was sufficient.

Most of the measurements reported were made using configuration $A$ for absorption and configuration $D$ for fluorescence. Configuration $B$ can have advantages over configuration $D$ in fluorescence for transparent preparations (like the salamander) because relatively large numerical aperture condensers can be used, increasing the illumination intensity. We used configuration $B$ in our initial testing of dyes but, because epi-illumination would be necessary for opaque preparations and convenient for in vivo preparations, most fluorescence experiments were done with configuration $D$. Configuration $C$ was used to test the possibility that absorption measured by reflection would give larger signals than would fluorescence.

A Wild $\times 7,0.2$ n.a. (numerical aperature), a Wild $\times$ $10,0.4$ n.a., and a Leitz $\times 32,0.6$ n.a., microscope objective was used. In our microscope the actual magnifications of these lenses were $\times 10.5, \times 15$, and $\times 31$, respectively.

Light from a tungsten filament bulb was passed through $30-\mathrm{nm}$ (width at half-height; except in Fig. 6, where the width at half-height was $90 \mathrm{~nm}$ ) interference filters (Filter 1) and focused on the preparation using Kohler illumination. For each dye, we tested several interference filters to find the wavelength that gave the largest signal-to-noise ratio. The output of each photo- detector was amplified twice and the amplified output was digitized and stored in a PDP 11/34 minicomputer. In most experiments each detector was sampled every 0.7 msec. For Figure 6 the rate was reduced to every 2 msec. To reduce noise, the high frequency response of the amplifiers was limited in two stages, each with a 0.4msec time constant. The low frequency response was limited by capacity coupling the two amplifiers; the coupling time constant was $100 \mathrm{msec}$. This coupling means that a long-lasting step increase in light intensity would be recorded as an abrupt increase followed by a fall to $63 \%$ of the way to the original level in $100 \mathrm{msec}$. Relatively long-lasting signals, e.g., Figure 2 traces 85 and 114 , are distorted by this coupling. The records shown in the figures were either cut-outs or tracings of the hard copy output from the computer. In some instances the individual data points were connected to improve clarity.

Dyes XVII (Ross et al., 1977) XXV (Gupta et al., 1981), RGA452 (the 3-carbethoxypyrazolone derivative of XXV), and RGA461 (the 3-ethylpyrazolone, di-pentyl barbituric acid derivative of XXV) were obtained from Dr. Alan Waggoner, Center for Fluorescence Research, Carnegie-Mellon University, Pittsburg, PA; dye XXII (NK2367) (Gupta et al., 1981) was from Nippon KankohShikiso Kenkyusho Co. Ltd., Okayama, Japan; and dyes RH160, RH237, RH292, RH364, and RH414 (Grinvald et al., 1982b) were from Dr. Amiram Grinvald, Department of Neurobiology, Weizmann Institute of Science, Rehovot, Israel.

Electrophysiology. Extracellular field potential recordings were made using micropipettes with resistances from 5 to 30 megohms. The potential recorded by the electrode was amplified and displayed on an oscilloscope or stored in the computer. The olfactory nerve was stimulated with a suction electrode. 
All experiments were carried out at room temperature, 20 to $23^{\circ} \mathrm{C}$.

\section{Results}

The transmission through the unstained olfactory bulb was measured in three preparations by comparing the light reaching the detectors when the preparation was in the light path $(I)$ and when it was removed $(I O)$. I/Io averaged $12 \%$, a value similar to that obtained from some molluscan ganglia (Boyle et al., 1983).

All of the dyes we tried had been tested previously on squid axons and had large signals on that preparation. Because past experience has shown that not all of the dyes which work well on squid will be satisfactory for a new preparation, we began by testing 15 dyes to see if they would stain the in vitro olfactory bulb. Staining was obtained with mosi of the dyes, but it was not always uniform. The styryl dye, $\mathrm{RH} 414$, at $1.0 \mathrm{mg} / \mathrm{ml}$, stained uniformly and penetrated throughout the bulb. On the other hand, a 10 -min exposure to $0.5 \mathrm{mg} / \mathrm{ml}$ of the merocyanine dye, XVII, stained the surface substantially more than the interior of the bulb.

From the staining experiments, 10 dyes were selected and were tested on in vitro preparations for absorption signals using transmitted light (Fig. $1 A$ ). Signals were obtained using 9 of the 10 dyes tested, namely: XVII, RGA461, XXII, RGA452, XXV, RH160, RH237, RH292, and $\mathrm{RH} 364$. Of these the merocyanine, XVII, seemed to give the largest signals. Seven dyes were tested for fluorescence signals; signals were found with dyes RGA461, RH160, RH292, RH364, and RII414. The styryls, RH160, and RH414, gave the largest signals.

An example of the absorption signals obtained from the in vitro bulb in response to suction electrode stimulation of the olfactory nerve is presented in Figure 2. This figure shows the simultaneously recorded changes in light intensity from a single trial measurement of transmission (Fig. 1A) using dye XVII. A sketch of the preparation as imaged onto the photodiode array is shown in the lower left; the olfactory nerve, entering from the upper left, was stimulated at the time indicated by the arrowhead on each trace. Dye XVII was used in seven additional in vitro preparations. Signal-to-noise ratios similar to those in Figure 2 were obtained in every experiment.

An examination of the signals on trace 85 (labeled in Fig. 2) and on neighboring traces suggests that threc rapid peaks and one slow signal may be distinguished, althought only a few traces contain all four signals and peaks merge in some traces. The earliest fast peak seen on detector 81 and other detectors receiving light from the region of the olfactory nerve is about $5 \mathrm{msec}$ in duration at half-height and reaches a peak 4 to $15 \mathrm{msec}$ from the time of the stimulus. This first peak propagates caudally (to the right in Fig. 2) at about $0.2 \mathrm{~m} / \mathrm{sec}$ and is presumed to originate from the action potentials in the olfactory nerve axons.

The second fast peak, seen as the largest peak in trace 85 and the one below it, is also about $5 \mathrm{msec}$ in duration (estimated above background) and reaches a maximum 11 to $18 \mathrm{msec}$ from the time of the stimulus. This peak propagates at about the same velocity but in the opposite direction. A preliminary speculation about the origin of the first two fast peaks is that they represent olfactory axon activity in two parts of the nerve axons which, due to the fortuitous position of the preparation, are both imaged on single elements of the array. The sketch in the lower right of Figure 2 illustrates how this might come about; light rays at some positions will pass through an individual axon at two places. Support for this suggestion comes from the finding that neither peak was suppressed in a double pulse experiment (see below). This kind of second peak was seen in only a few preparations.

The third fast peak, seen as the largest peak in trace 114 , is about $7 \mathrm{msec}$ in duration and peaks 23 to $35 \mathrm{msec}$ from the time of the stimulus. Its latency is similar to that of the postsynaptic activity of mitral, tufted, and periglomerular cells in the rabbit and turtle (Shepherd, 1963; Waldow et al., 1981) and is likely to be a postsynaptic response (see below). A peak with a similar latency was seen in seven other experiments.

The slower signals, seen on all of the detectors except those receiving light from the olfactory nerve, have a width at half-height of from 50 to more than $100 \mathrm{msec}$. They reach a maximum from 35 to at least $80 \mathrm{msec}$ from the time of the stimulus. The time to peak increases but the duration decreases as the signal proceeds caudally. The shape of these slow signals is more or less distorted by the $100-$ msec coupling time constant of the photodiode amplifiers, which will cause the signals to return to base line too rapidly. Slow signals were seen in almost all experiments.

The largest change in intensity, divided by the resting intensity, was measured for trace 87 (below trace 81) in the experiment illustrated and was $5 \times 10^{-3}$. Values between 1 and $5 \times 10^{-3}$ were obtained in other traces of this experiment. These fractional changes are relatively large, about 10 times larger than those found in squid axon experiments with the same dye (Ross et al., 1977). One explanation that could account for the larger fractional change in the salamander experiments is that each light ray passed through more active membrane; i.e., more cells in a light path were simultaneously active in the salamander measurement as opposed to the squid measurement. Absorption signals were also found with dye XVII using epi-illumination with a quarter-silvered mirror (Fig. $1 C$ ). The signal-to-noise ratios were substantially smaller than those illustrated in Figure 2 and smaller than fluorescence signals measured with epiillumination (see below), probably because the light levels were low.

Controls. Before presenting additional results, we want to show that the changes in light intensity in Figure 2 were not caused by events other than changes in membrane potential and that the intensity changes are not stimulus artifacts. The possibility of a direct coupling between the electrical measuring system and the light measuring system can be ruled out because no signals were obtained with the light off. The possibility that the signals result from light scattering changes (Cohen, 1973) or mechanical artifacts may be rejected for three reasons. First, no signals were found before staining; second, no signals were found at wavelengths outside the absorption 


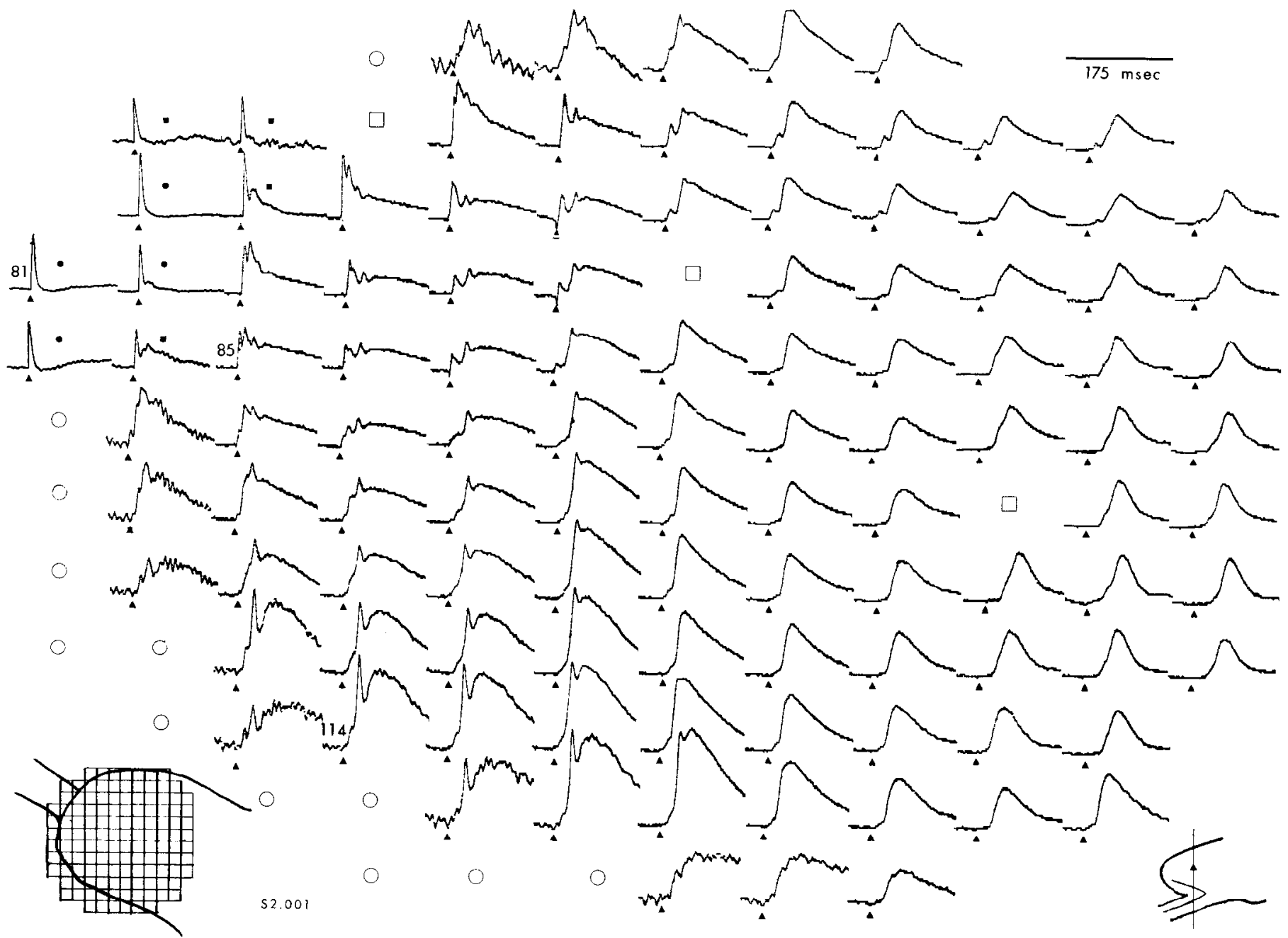

Figure 2. Absorption signals (Fig. 1A) simultaneously measured by 111 diodes from an in vitro salamander olfactory bulb. An increase in intensity reaching the photodiodes is drawn upward. In this figure and in Figure 6 the traces are arranged so that their relative positions in the figure correspond to the relative positions of the areas of the preparation imaged onto the detectors. The inset on the lower left illustrates the location of the image of the bulb on the array elements. The olfactory nerve, imaged on the upper left portion of the array, was stimulated via suction electrode with a 0.2 -msec pulse of $2 \mathrm{~mA}$ at the time indicated by the arrowheads on each trace. Three sharp peaks and slower signals corresponding to electrical activity can be seen. Three of the amplifiers were not functioning; these array elements are indicated by the open squares. Eleven of the elements, indicated by open circles, did not have signals; these detectors received light that did not pass through the preparation. All of the traces were recorded at the same amplifier and computer gains except for eight traces in the upper left-hand corner. The traces with solid squares were reduced in size by a factor of 2 ; the traces with solid circles were reduced by a factor of 4 . The resting light intensity reaching detector 87 (below 81 ) was about $4 \cdot 10^{9}$ photons $/ \mathrm{msec}$. The bulb had been stained in $0.5 \mathrm{mg} / \mathrm{mI}$ of dye XVII for $10 \mathrm{~min}$ and then rinsed. The image of the bulb was projected on the detector array with a Wild $\times 7,0.2$ n.a. objective; the condenser was set for Kohler illumination. Each detector received light from a $133 \mu \mathrm{m}^{2}$ area of the bulb. Filter 1 had a peak transmission at 750 $\mathrm{nm}$.

band of the dye; and third, the dye-related signals have a complex wavelength dependence. This is in contrast to light scattering signals which are monotonically decreasing with increasing wavelength and which exist at all visible wavelengths (Cohen and Keynes, 1971). Figure 3 illustrates the wavelength dependence for the oxonol dye, RGA452. This figure shows the output of a single detector for different wavelengths of incident light in an absorption measurement. At $600 \mathrm{~nm}$ all detectors had signals which were an increase in intensity; at $660 \mathrm{~nm}$ all detectors had signals which were a decrease. The fast and the slow signals had the same wavelength dependence, reversing sign between 600 and $630 \mathrm{~nm}$. No signals were found at $720 \mathrm{~nm}$. With the merocyanine dye, XVII, there were two sign reversals in the visible spectrum. Complex wavelength dependencies of this sort are very similar to results obtained in squid axons (Ross et al., 1977; Gupta et al., 1981) and are the expected result if a potential change causes a wavelength shift in the absorption spectrum. Because the direction of the optical signal resulting from a depolarization depends on wavelength for a given dye and is different for different dyes, the direction of the signal cannot be used to determine the sign of the potential change. However, since stimulation of the olfactory nerve will always cause a depolarization, the direction of the signal obtained from the nerve pro- 
vides a calibration. We have drawn this signal upward in all figures (except Fig. 3).

Presumably, activity-dependent light scattering signals must be present inasmuch as they have been detected in many preparations (Cohen, 1973) including brain slices (Grinvald et al., 1982a). However, these kinds of scattering signals were small in other preparations and thus might be difficult to detect at the amplifier gains used for present experiments. We conclude that the signals have a wavelength dependence that is expected if they represent changes in membrane potential. Although our present evidence is consistent with the conclusion that the signals from the bulb itself, reported in this paper, do not result from light scattering, we did find a wavelength-independent, dye-independent signal

WAVELENGTH DEPENDENCE

$510 \mathrm{~nm}$

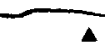

$600 \mathrm{~nm}$

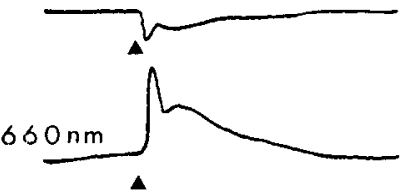

$720 \mathrm{~nm}$

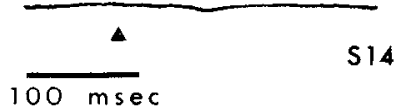

Figure 3. Wavelength dependence of absorption signals (Fig. 1A) from an in vitro olfactory bulb stained with $0.05 \mathrm{mg} / \mathrm{ml}$ of RGA452 for $10 \mathrm{~min}$. The olfactory nerve was stimulated at the time indicated by the triangles. The signal was small for short wavelengths, reversed sign at 600 to $630 \mathrm{~nm}$, reached a maximum at $660 \mathrm{~nm}$, and was small at longer wavelengths. An increase in intensity reaching the photodiodes is drawn downward. The olfactory nerve was stimulated via suction electrode with a 0.4 -msec pulse. A Leitz $\times 32,0.6$ n.a. objective with a 0.45 n.a. condenser was used. These traces were redrawn with high frequency noise smoothed. in three measurements from the caudal region of the cerebral hemispheres. The origin of this signal is not known.

Additional experiments indicate that none of the signals detectable at the gain used (Fig. 2) results from passive spread of potential from axon membrane that was directly polarized by the stimulating electrode. First, the signals did not reverse in sign when stimulus polarity was reversed. Second, in another experiment, illustrated in Figure 4, the optical signals were abolished by tetrodotoxin (TTX). There was a partial recovery of the signals after the TTX was rinsed off (not shown). In other experiments of this type, there was a relatively small, rapidly occurring signal that remained after the addition of TTX. This small signal could either result from passive spread of potential from the stimulated region or be a stimulus artifact.

Pharmacology. We did not do a systematic study of pharmacologic or photodynamic effects of the dyes on the olfactory bulb. Incubation with dye XVII $(0.5 \mathrm{mg} /$ $\mathrm{ml})$ and RH160 $(0.02$ and $0.1 \mathrm{mg} / \mathrm{ml})$ led to increases in both the time to peak and the duration of components of the field potentials; however, both the time to peak and the duration returned toward their original values after replacing the staining solution with Ringer's.

Paired volley experiment. To determine whether the signals in Figure 2 arose from olfactory nerve axons (presynaptic) or from neurons in the bulb (postsynaptic) we have relied on the demonstration, by Waldow et al. (1981), that the first stimulation in a paired volley experiment will suppress postsynaptic activity elicited by the second stimulus. Thus, components of the signals in Figure 2 that reflect postsynaptic activity should be suppressed by a prior stimulus. The result of such an experiment, using the same bulb as in Figure 2, is shown in Figure 5; the figure shows four representative optical records and a field potential record. Both optical and electrode recordings show selective suppression of the signals occurring at longer latencies. The first two peaks, in traces 81 and 91 , are not affected by the conditioning pulse, whereas the third peak and slow components in traces 91,117 , and 27 are markedly suppressed. The results of this experiment are consistent with the possibility suggested above that the first and second peaks are

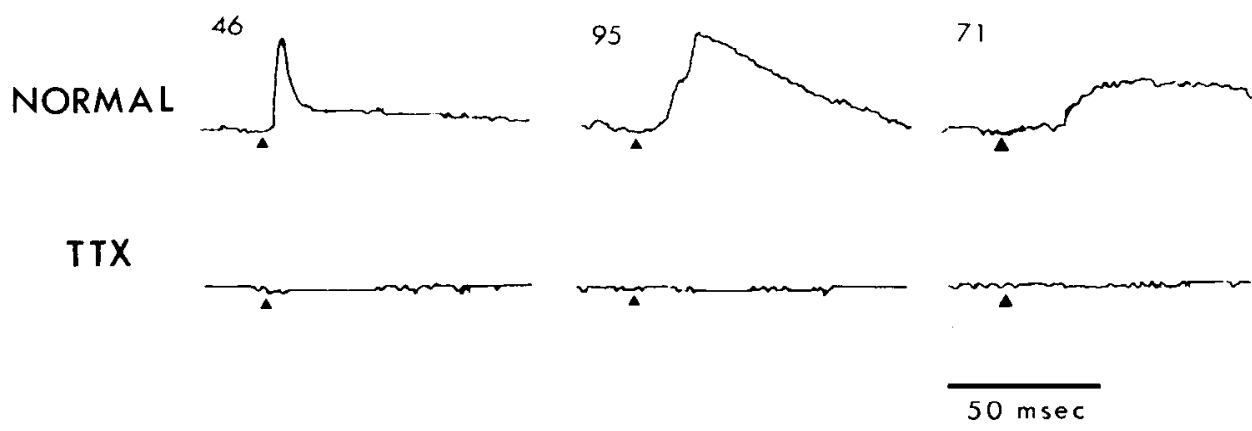

Figure 4. Effects of $10 \mu \mathrm{M}$ TTX on the optical signals. Traces from three representative detectors are shown. The first row illustrates fluorescence signals (Fig. $1 D$ ) from the three detectors before application of TTX. The second row shows the photodiode outputs, using the same stimulus, after the preparation was incubated in TTX for 7 min. TTX blocked all signals. A decrease in intensity reaching the photodiodes is drawn upward. This in vitro olfactory bulb had been dyed for 10 min with dye $\mathrm{RH} 160$. The olfactory nerve was stimulated at the time indicated by the triangles. No incident light filter (Filter 1 , Fig. $1 D$ ) was used. Filter 2 was an 0G590 filter (Schott Optical Glass, Duryea, PA). A Wild × 10, 0.4 n.a. objective was used. 


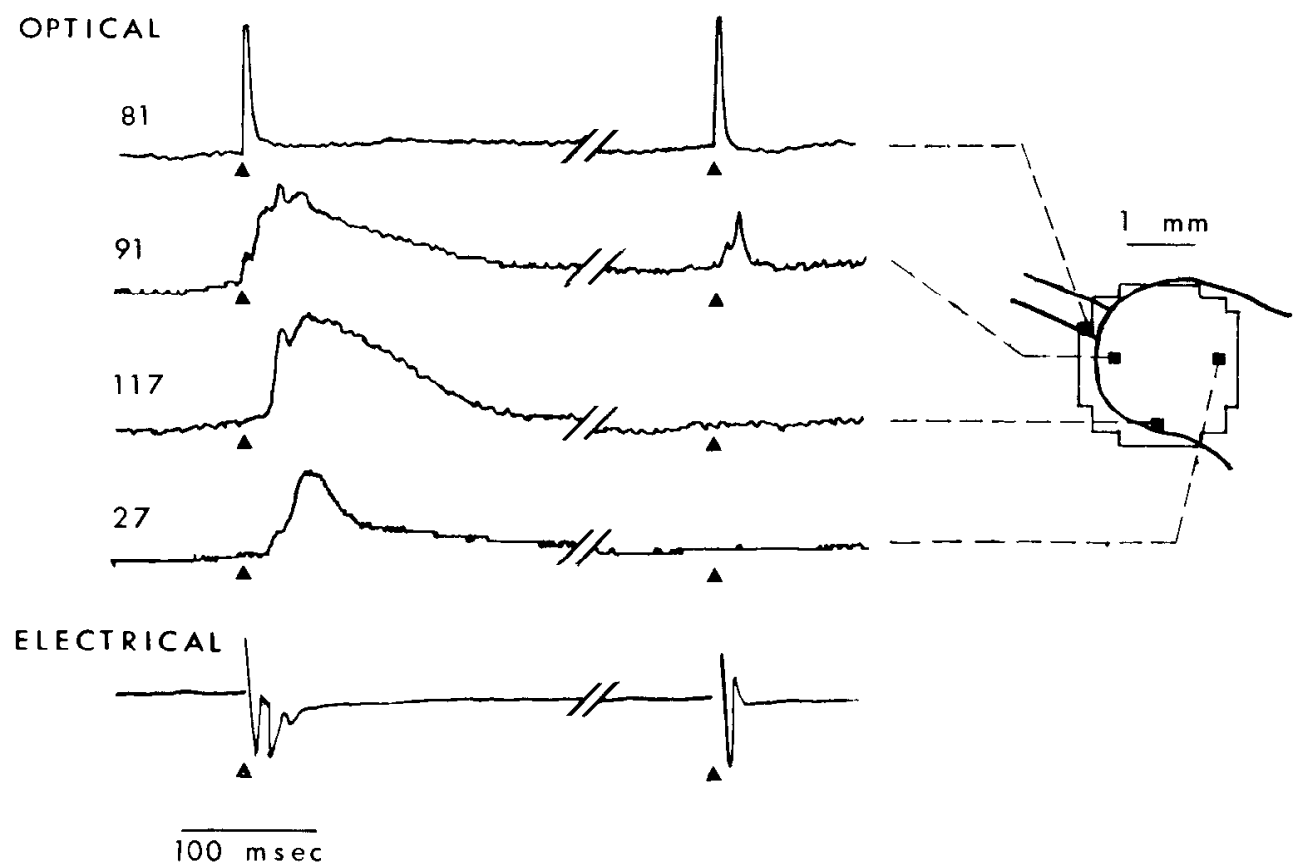

Figure 5. A paired volley experiment. Absorption signals (Fig. 1A) identified with postsynaptic activity are suppressed by the conditioning pulse; peaks identified with presynaptic olfactory nerve activity show no suppression. The top four traces are representative optical traces; the bottom trace is a simultaneous microelectrode recording of field potentials. There was a 600 msec delay between the conditioning and the test pulse. These results were obtained from the same preparation used for the experiments shown in Figure 2. An increase in intensity reaching the photodetectors is drawn upward.

due to the compound action potential in the olfactory axons and that the third sharp peak and slow signals are postsynaptic responses.

This same dramatic suppression of the third peak and slow signals was seen on all of the detectors in this trial and other trials in this experiment. However, in other experiments the suppression of the slow components in the second response was less complete.

In vivo experiments. Whereas the preceding results showed that relatively large signal-to-noise ratios could be obtained from in vitro preparations, the circulation of blood in the in vivo preparation could lead to movement signals which would obscure the dye changes. Even though hemoglobin is not very fluorescent, it does absorb at the wavelengths ( 510 and $540 \mathrm{~nm}$ ) used for excitation of the styryl dyes. For the in vivo experiment, the top of the skull was removed from midway along the telencephalic hemispheres forward, and the salamander was put on the stage of the microscope. Again, a suction electrode was used to stimulate a cut olfactory nerve. Signals from in vivo bulbs were obtained using both transmission absorption (not shown) and epi-illumination, using the fluorescence of dyes RH160 and RH414. The traces in Figure 6 illustrate the outputs of a portion of the diode array from an experiment using $\mathrm{RH} 414$. In this experiment the olfactory nerve entered the field of view from the upper right. The signals measure from the in vivo bulb had sizes and time courses similar to those measured in vitro. The in vivo measurements (Fig. 6) did have somewhat more low frequency noise than the in vitro results, but the signal-to-noise ratio remains relatively large in single trials. RH414 was recently synthesized by $R$. Hildesheim and A. Grinvald and was first used in experiments on rat cortex (Orbach et al., 1983).
The traces shown in Figure 6 were scaled by dividing the changes in intensity by the resting fluorescence at each detector. Thus the size of the signal in each trace represents the relative size of the fractional change in fluorescence, $\Delta F / F$, rather than $\Delta I$ or $\Delta F$ as in Figures 2 to 5 . In the experiment shown in Figure 6 the peak $\Delta F / F$ was $1.3 \cdot 10^{-2}$. In a second in vivo experiment with the same dye the peak $\Delta F / F$ was $3 \cdot 10^{-2}$, and in an in vitro experiment with dye $\mathrm{RH} 160$ the $\Delta F / F$ was $5 \cdot 10^{-2}$. As was the case with the absorption signals, these fractional changes are relatively large, about 10 times those found in experiments with the same dyes on squid axons (Gupta et al. 1981; A. Grinvald, R. Hildesheim, and L. B. Cohen, unpublished results). One possible explanation for the larger fractional fluorescence change in the salamander is that a larger proportion of the membrane present in the preparation is active. In the squid axon preparation the area of the nonactive membrane (Schwann cell and connective tissue cells) is more than 10 times larger than the area of axon membrane (Binstock et al., 1975). The large fractional change in fluorescence in Figure 6 helps to explain why there was relatively little interference from the clearly visible blood flow.

Limitations on spatial resolution. We wanted to obtain an estimate of the limits on the resolution in the $X-Y$ plane due to light scattering and due to the broadening of discrete signals when their source is not in focus. Starting with a $40-\mu \mathrm{m}$ spot of light we measured the intensities imaged on elements of the array with the $x$ 10 objective. For the experiments illustrated in Figure 7 the spot was illuminated for a $200-\mathrm{msec}$ interval during a 600-msec recording; the intensity reaching each detector is indicated by the height of the step in each trace. A 


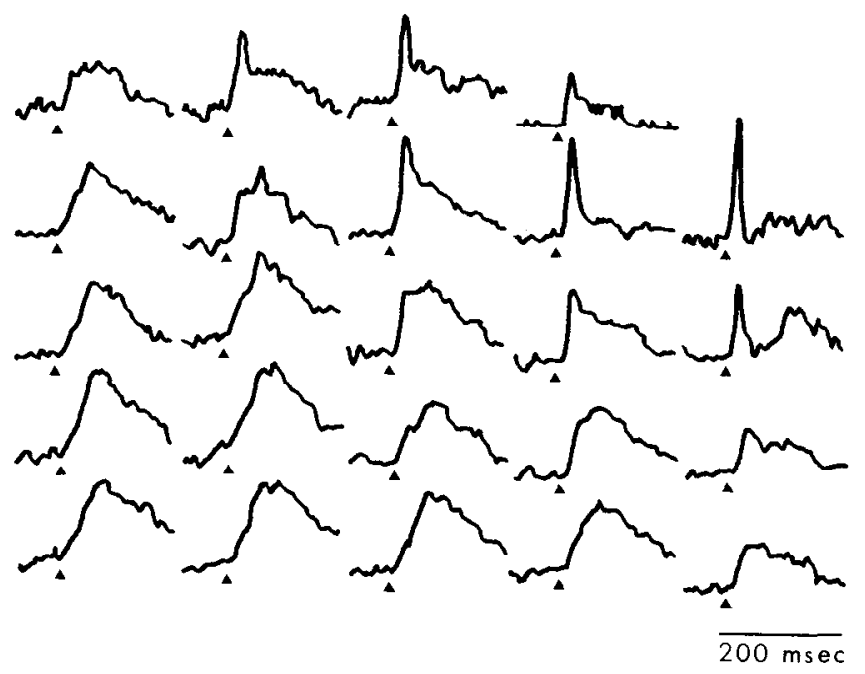

39.001

Figure 6. Fluorescence measurements from 25 detectors from an in vivo salamander olfactory bulb. The olfactory nerve was stimulated with a suction electrode using a $0.3-\mathrm{msec}$ pulse at the times indicated by the triangles. The brain had been stained with $1.5 \mathrm{mg} / \mathrm{ml}$ of $\mathrm{RH} 414$ perfused through the cranial cavity for 75 min. A $\times 7,0.2$ n.a. objective was used; an area of 133 $\mu \mathrm{m}^{2}$ was projected on each detector. The measurement was of fluorescence using epi-illumination (Fig. $1 D$ ). The resting light intensity reaching the photodetectors was about $7 \cdot 10^{7}$ photons/ msec, approximately 100 times less than the resting intensity in absorption measurements. A decrease in intensity reaching the photodetectors is drawn upward. A 520-nm incident light filter (Filter 1) was used with an RG610 post-filter (Filter 2). In addition to the high frequency filtering done by the amplifiers, we used three passes of a 1-2-1 digital smoothing routine before displaying the traces. At the sampling rate used (one sample each $2.0 \mathrm{msec}$ ), this additional filtering will be approximately equivalent to three R-C filters with time constants of 2 msec.

$5 \times 7$ element portion of the array is illustrated in each panel of the figure.

When the $40-\mu \mathrm{m}$ spot was in focus and only saline and air were between the spot and the objective lens, essentially all of the light from the spot fell on a single detector (Fig. $7 A$ ). In Figure $7 B$, the result of moving out of focus by $500 \mu \mathrm{m}$ is illustrated. In this case the light is approximately equally distributed on the original detector and on four of its neighbors. In a measurement with a 1000 $\mu \mathrm{m}$-thick olfactory bulb and the $\times 10$ objective focused at the middle of the bulb, the average error in focus will be only $250 \mu \mathrm{m}$. Thus, the broadening due to out-of-focus signals will, on average, be half that shown in Figure $7 B$. In Figure $7 C$ the spot was again in focus, but a $500-\mu \mathrm{m}$ thick slab of salamander cortex was positioned between the pinhole and the objective lens. Again, the image of the spot is spread over several detectors, although the pattern is not precisely the same as that obtained with the out-of-focus spot. With the $\times 10$ objective used in this trial the centers of the individual detectors are measuring light from regions of the plane of focus that are $100 \mu \mathrm{m}$ apart. The diameter of the circle of light that includes intensities of greater than $50 \%$ of the maximum was approximately two detector widths or $200 \mu \mathrm{m}$. The percentage of the light that was scattered away from the
A. PINHOLE IN FOCUS

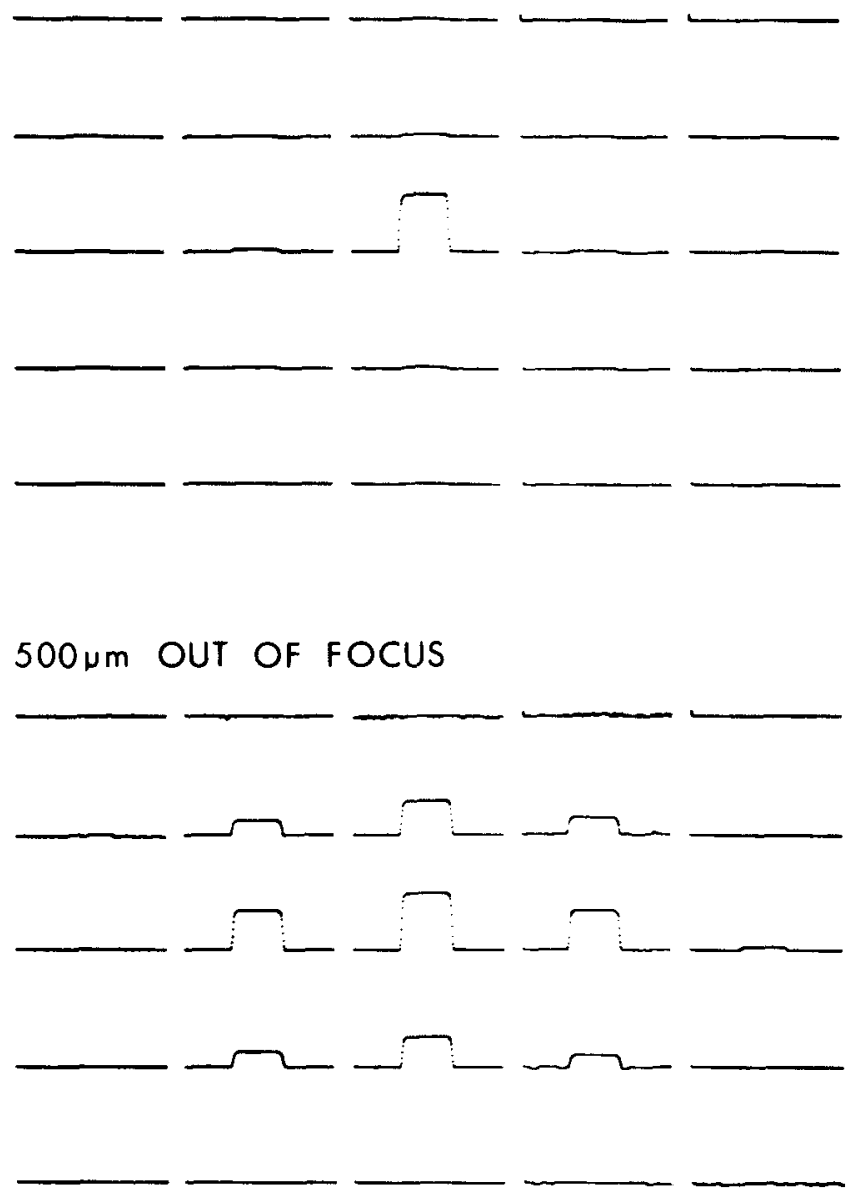

\section{B. $500 \mu \mathrm{m}$ OUT OF FOCUS}

\section{IN FOCUS UNDER $500 \mu \mathrm{m}$ OF CORTEX}

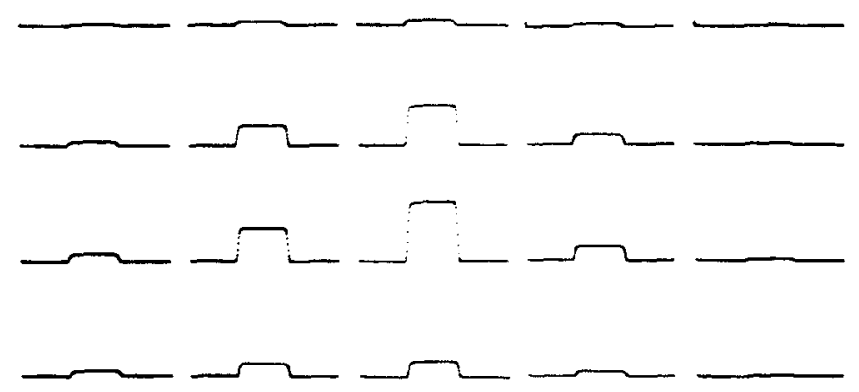

Figure 7. Fffects of focus and scattering on the distribution of light from a point source onto the array. In $A$, a $40-\mu \mathrm{m}$ pinhole in aluminum foil covered with saline was illuminated. The pinhole was in focus. More than $90 \%$ of the light fell on one detector. In $B$, the stage was moved downward by $500 \mu \mathrm{m}$. I ight from the out-of-focus pinhole was now seen on several detectors. In $C$, the pinhole was in focus but covered by a 500 $\mu \mathrm{m}$-thick slab of salamander cortex. The light from the pinhole was spread over several detectors. $\mathrm{A} \times 10,0.4 \mathrm{n}$.a. objective was used. Before the pinhole was placed in the object plane the optics were set up using Kohler illumination. The recording gains were adjusted so that the largest signal in each of the three trials would be approximately the same size in the figure. 
central detector was less with objectives of lower magnification and greater with objectives of higher magnification. It was difficult to detect scattering in measurements from the same preparation when a $\times 3.5$ lens was used.

\section{Discussion}

Our purpose was to detect optically the electrical activity in a favorable CNS preparation with the ultimate objective of using the optical technique to monitor activity in response to physiological stimuli in both lower vertebrate and mammalian preparations. Below we argue that the signals we found are large enough to accomplish this objective.

Although the signals were large in most experiments, comparison of the results in Figures 2 to 6 shows that the amount of detail in the signals varied from experiment to experiment. We do not know the origin of these differences. Among many possibilities are differences in staining or orientation of the bulb. Because we did not control the orientation of the bulb and did not do systematic recordings of field potential, a detailed determination of the relationship of the optical signals to olfactory bulb anatomy and physiology will require additional experiments. However, it is possible that the rapid postsynaptic signal represents mitral cell spikes and that the slow postsynaptic signal seen in caudal regions represents granule cell excitatory potentials (Rall and Shepherd, 1968).

Even though optical methods do have the advantages mentioned in the introduction, they also have limitations and caution must be exercised in interpreting the signals. One limitation is that it will be difficult to detect signals from regions that are deep within a preparation. A bulb, approximately $1 \mathrm{~mm}$ thick, had approximately $10 \%$ transmittance after staining with dye XVII, and absorption signals were easily recorded through this preparation. However, using transmitted light in a thicker preparation with the same opacity per millimeter, the light reaching the detectors would decrease by a factor of 10/ $\mathrm{mm}$. Assuming a shot-noise limited measurement, the signal-to-noise ratio would decrease as the square root of intensity or by $\sqrt{10 / \mathrm{mm}}$. For epi-illumination (Fig. 1 , $C$ and $D$ ), which may be necessary for large, more opaque brains, signals would decrease even more rapidly with depth below the surface. Assuming a $10 \%$ transmittance/ $\mathrm{mm}$, than the light reaching the detectors from a given depth will decrease by a factor of $10^{2} / \mathrm{mm}$ resulting in a decrease in signal-to-noise ratio of $10^{1} / \mathrm{mm}$. Thus, it seems clear that with present techniques in situ measurements from deep structures will be difficult and would require more sophisticated methods for staining and illumination. This calculation suggests that for interior structures in opaque preparations, transmission measurements would in principle give larger signals than epiillumination. However, in situations where dye binding (or neuronal activity) is restricted to the outer brain layers, epi-illumination could be preferable. Furthermore, signals will be difficult to obtain from surfaces (such as sulci or surgically inaccessible areas) whose image cannol be projected on the detector array. These problems are alleviated if animals with smaller brains are used.
A second difficulty with optical methods lies in disentangling the signals from different depths. However, two factors may aid in determining the depth at which a signal originated. First, when epi-illumination is used, opacity will reduce signals from greater depths. Second, it is possible that signals from isolated discrete structures, covering a small part of the field, can be dissected to some degree by optical sectioning; signals from structures out of focus will be smaller than those from structures in focus. In measurements on barnacle ganglia, Salzberg et al. (1977) found that changing the focus by $300 \mu \mathrm{m}$ led to a $50 \%$ reduction of signal size using a 0.4 n.a. objective. With lenses of larger numerical aperture, increased sensitivity to focal position would be obtained. In preliminary olfactory bulb experiments we found that changing the focus had only a small effect on signal size or shape. This may, however, be the expected result considering the orientation of the cell layers in the salamander bulb (Herrick, 1965).

There are also limitations in resolution in the plane of focus. With an array of only 124 elements filling the image plane, the finite size of each detector means that the light from many neurons will reach each detector. With the $\times 7, \times 10$, and $\times 32$ objectives, object areas of $133 \mu \mathrm{m}^{2}, 94 \mu \mathrm{m}^{2}$, and $45 \mu \mathrm{m}^{2}$ respectively, project onto each detector element. A further limitation on resolution occurs because light scattering by the tissue will blur the images formed at the photodiode array. Light scattering will cause a signal which originates from a small volume of the brain to appear over a larger area of the array than one would predict from the magnification of the objective lens (Fig. $7 \mathrm{C}$ ). That result combined with the results in Figure 2, where, in many instances the time courses of the signals in adjacent detectors are significantly different, indicates a resolution of approximately $200 \mu \mathrm{m}$ in a uniformly stained salamander olfactory bulb. The limitations on resolution from light scattering and out-of-focus signals would be relieved if the staining were restricted to a thin cortex of the preparation. Thus, although our results support the suggestion that optical methods have better spatial resolution than do presently available tomographic techniques, it seems that the spatial resolution of an optical measurement will not be as good as that which can be obtained in 2deoxyglucose or cytochrome oxidase measurements.

With present apparatus, it does not seem possible to determine the origin of the signals at the level of individual cells using extracellularly applied dyes that bind relatively indiscriminately to all cell membranes. Intracellular administration of dyes, or dyes with cytologically specific binding, might allow a direct determination of the cellular origins of optical signals.

It will be difficult to determine the average membrane potential change from the recorded optical signals and it will be difficult to compare the magnitudes of signals on different detectors in a meaningful way. One might think that each detector's signal represents the (average of change in membrane potential) $\times$ (membrane area) imaged on the detector and thus that signals from different detectors could be compared quantitatively once membrane area was known. However, not only is the measurement of membrane area difficult, but several other factors are also important in determining signal size. 
These include the dye access to membranes and the relative amount of dye bound to neuronal and nonneuronal membrane. The dye access and dye binding to different cell types is unlikely to be constant and is probably complex; for example, it is known that the relationship between staining density and signal size is nonlinear in several preparations (Cohen et al., 1974, Grinvald et al., 1981b).

The assignment of the slow signals to changes in membrane potential may be less certain than for the fast signals. Beeler et al. (1981) found that relatively large changes in divalent ion concentration led to changes in the fluorescence of dye XXV in sarcoplasmic reticulum suspensions. This raises the possibility that, for example, $\mathrm{K}^{+}$accumulation in the extracellular space might lead to slow signals by affecting dye binding to membranes. One argument against such a possibility is the absence of slow signals on detectors receiving light solely from olfactory axons or their terminals. Furthermore, the fact that the fast and slow signals have identical wavelength dependences (Fig. 3) is consistent with the notion that both signals reflect changes in membrane potential. Finally, slow potential changes are expected since there are very long-lasting effects of single stimulations in the olfactory bulb (Waldow et al., 1981; Fig. 5).

Our measurements of the slow signals were not accurate because of distortion introducted by the 100 -msec coupling time constant of the diode amplifiers. Furthermore, although experiments with long-lasting potential changes carried out on other preparations (Ross et al., 1977; Gupta et al., 1981; Ross and Krauthamer, 1983) have shown that the dye signals at long times are potential dependent; in some instances the dye signal does not follow potential in a simple way. Because they were found with many different dyes, we are confident about the existence of slow signals in the olfactory bulb; however, accurate determination of their time course requires additional experiments.

A comparison of optical measurements of activity with field potential measurements suggests that the two may provide very different but complementary information. Whereas the optical measurement is, as far as is known, strictly determined by membrane potential, the signal recorded in a field potential measurement has a complex relationship to the membrane potential of the active cell population and to the position of the recording electrodes (Rall and Shepherd, 1968; Llinás and Nicholson, 1974). The extracellular potential may be related to either membrane potential or, more often, to its first or second derivative. Thus, one field potential in the bulb changes sign as the electrode is advanced past the mitral cell layer, a kind of result that will not be found using techniques (including optical measurements) that measure membrane potential. The signal size of an optical measurement will, in general, be proportional to the membrane area of the cells imaged on the detector, whereas the field potential measurement will give a result that is proportional to the cross-sectional area of a process and is thus more biased toward large processes and neurons. The optical signal will be nearly insensitive to neuronal geometry or orientation, whereas a field poten- tial measurement is very sensitive to these factors. Finally, except for the blurring due to light scattering and out-of-focus regions, the spatial origins of optical signals are well defined, whereas the origin of a field potential signal can be located only with difficulty. Thus, in many respects the difficulties of interpreting signals from voltage-sensitive dyes do not overlap the difficulties in interpreting field potential measurements. Similarly, possible pharmacologic effects of the dye are not likely to lead to the same kind of damage as electrode penetration into the brain.

One potential advantage of an optical method is that one can increase the number of simultaneous recordings beyond the 124 used in the experiments reported here. Charge-coupled device (CCD) arrays and other imaging devices with more than $10^{5}$ elements (pixels) are commercially available. Because of considerations of signal size and blurring of the image due to light scattering, it is not clear what the useful lower limit of brain area imaged onto an individual detector will be. This lower limit may determine the upper limit to the useful number of array elements.

We think that the techniques demonstrated here could be used to provide a real-time map of brain activity in many situations. If input and output signals can be identified, then the optical measurements could also provide data about the information processing in the regions under investigation. Because we have demonstrated that signals can be obtained from salamander olfactory bulb, one possibility would be to use the signals to explore the functional organization of the olfactory system. This might be done using physiological stimulation techniques such as those of Kauer (1974) to study the functional identity of glomeruli or to locate where excitation from individual odors projects in the bulb. This would allow a comparison of the results from an optical measurement with the results already obtained using 2-deoxyglucose (Stewart et al., 1979; Lancet et al., 1982). We expect that the technique, as already developed, could be used in fish, amphibians, and reptiles as an alternative method to staining or field potential measurements to map projections of sensory systems or nerve tracts. This seems to be the case. Grinvald et al. (1983) recently reported optical signals in frog optic tectum in response to light stimuli to the eye.

Optical methods may also be applicable to the mammalian CNS. Exploratory experiments to determine which dyes penetrate and stain rat cortex have been carried out and signals were detected from visual cortex in response to visual stimulation (Orbach et al., 1982) and from somatosensory cortex in response to whisker movement (Orbach et al., 1983). We are hopeful that these methods may be applicable at the level of a gross mapping of cortical areas, where, for example, primary and secondary areas can be monitored simultaneously; it might also be possible to monitor activity from individual cortical columns. Optical measurements might also be useful in the study of the spread of seizure activity. In this kind of experiment, the percentage of involved cells is large and thus the signals should be large.

On the other hand, it is likely that physiological stim- 
ulation will activate a smaller fraction of cells than was achieved in the olfactory bulb by stimulation of the olfactory nerve. Although smaller signals are expected, several techniques for improving the signal-to-noise ratio are available. Since the signals we measured in the bulb were large, signal-averaging techniques were not used. But these computer programs are available for the diode array, and averaging 100 trials would increase the signalto-noise ratio by a factor of 10 . Thus, the smallest detectable signal would be at least 100 times smaller than those seen in these experiments; i.e., from $1 \%$ of the fraction of cells stimulated in these experiments, or from activity from deeper structures. Additional increases in signal-to-noise ratio could be obtained by using a more intense light source.

\section{References}

Aubert, X., B. Chance, and R. O. Keynes (1964) Optical studies of biochemical events in the electric organ of Electrophorus. Proc. R. Soc. Lond. Biol. 160: 211-245.

Beeler, T. J., R. H. Farmen, and A. N. Martinosi (1981) The mechanism of voltage-sensitive dye responses on sarcoplasmic reticulum. J. Membr. Biol. 62: 113-137.

Binstock, L., W. J. Adelman, Jr., J. P. Senft, and H. Lecar (1975) Determination of the resistance in series with the membranes of giant axons. J. Membr. Biol. 21: 25-47.

Boyle, M. B., and L. B. Cohen (1980) Birefringence signals that monitor membrane potential in cell bodies of molluscan neurons. Fed. Proc. 39: 2130.

Boyle, M. B., L. B. Cohen, E. R. Macagno, and H. S. Orbach (1983) The number and size of neurons in the CNS of gastropod molluses and their suitability for optical recordings of activity. Brain Res. 266: 305-317.

Cohen, L. B. (1973) Changes in neuron structure during action potential propagation and synaptic transmission. Physiol. Rev. 53: 373-418.

Cohen, L. B., and R. D. Keynes (1971) Changes in light scattering associated with the action potential in crab nerves. J. Physiol. (Lond.) 212: 259-275.

Cohen, L. B., and B. M. Salzberg (1978) Optical measurement of membrane potential. Rev. Physiol. Biochem. Pharmacol. 83: $35-88$.

Cohen, L. B., B. M. Salzberg, H. V. Davila, W. N. Ross, D. Landowne, A. S. Waggoner, and C. H. Wang (1974) Changes in axon fluorescence during activity: Molecular probes of membrane potential. J. Membr. Biol. 19: 1-36.

Grinvald, A., L. Anglister, R. Hildesheim, and J. A. Freeman (1983) Optical monitoring of naturally evoked dynamic patterns of neural activity from the intact frog optic tectum using a photodiode array. Soc. Neurosci. Abstr., in press.

Grinvald, A., W. N. Ross, and I. Farber (1981a) Simultaneous optical measurements of electrical activity from multiple sites on processes of cultured neurons. Proc. Natl. Acad. Sci. U. S. A. 78: $3245-3249$.

Grinvald, A., L. B. Cohen, S. Lesher, and M. B. Boyle (1981h) Simultaneous optical monitoring of activity of many neurons in invertebrate ganglia using a 124 element photodiode array. J. Neurophysiol. 45: 829-840.

Grinvald, A., A. Manker, and M. Segal (1982a) Visualization of the spread of electrical activity in rat hippocampal slices by voltage-sensitive optical probes. J. Physiol. (Lond.) 333: 269-291.

Grinvald, A., R. Hildesheim, I. C. Farber, and L. Anglister (1982b) Improved fluorescent probes for the measurement of rapid changes in membrane potential. Biophys. J. 39: 301308.
Gupta, R. K., B. M. Salzberg, A. Grinvald, L. B. Cohen, K. Kamino, S. Lesher, M. B. Boyle, A. S. Waggoner, and C. H. Wang (1981) Improvements in optical methods for measuring rapid changes in membrane potential. J. Membr. Biol. 58: 123-137.

Herrick, C. J. (1965) The Brain of the Tiger Salamander, p. 382, The University of Chicago Press, Chicago.

Kauer, J. S. (1974) Response patterns of amphibian olfactory bulb neurones to odour stimulation. J. Physiol. (Lond.) 243: 695-715.

Kaufman, L., and S. J. Williamson (1982) Magnetic location of cortical activity. Ann. N. Y. Acad. Sci. 388: 197-213.

Krauthamer, V., and W. N. Ross (1982) Dendrites of a barnacle neuron are excitable: Determination with voltage sensitive dyes. Soc. Neurosci. Abstr. 8: 685.

Krauthamer, V., and W. N. Ross (1983) Regional variations in excitability of barnacle neurons. J. Neurosci., in press.

Lancet, D., C. A. Greer, J. S. Kauer, and G. M. Shepherd (1982) Mapping of odor-related neuronal activity in the olfactory bulb by high-resolution 2-deoxyglucose autoradiography. Proc. Natl. Acad. Sci. U. S. A. 79: 670-674.

Llinás, R., and C. Nicholson (1974) Analysis of field-potentials in the central nervous system. In Handbook of Electroencephalography and Clinicial Neurophysiology, C. F. Stevens, ed., Vol. 2, Part B, pp. 61-92.

Mayevsky, A., N. Zarchin, and C. M. Friedli (1982). Factors affecting the oxygen balance in the awake cerebral cortex exposed to spreading depression. Brain Res. 236: 93-105.

Mori, K., and G. M. Shepherd (1979) Synaptic excitation and long-lasting inhibition of mitral cells in the in vitro turtle olfactory bulb. Brain Res. 172: 155159.

Orbach, H. S., and L. B. Cohen (1982) Simultaneous optical monitoring of activity from many areas of the salamander olfactory bulb: A new method for studying functional organization in the vertebrate CNS. Soc. Neurosci. Abstr. 8: 936.

Orbach, H. S., L. B. Cohen, and A. Grinvald (1982) Optical monitoring of evoked activity in the visual cortex of the marine rat. Biol. Bull. 163: 389 .

Orbach, H. S., L. B. Cohen, A. Grinvald, and K. Hildesheim (1983) Optical monitoring of neuron activity in rat somatosensory and visual cortex. Soc. Neurosci. Abstr., in press.

Raichle, M. E. (1979) Quantitative in vivo autoradiography with positron emission tomography. Brain Res. Rev. 1: 4768.

Rall, W., and G. M. Shepherd (1968) Theoretical reconstruction of field potentials and dendrodendritic synaptic interactions in olfactory bulb. J. Neurophysiol. 31: 884-915.

Ross, W. N., and V. Krauthamer (1983) Optical measurements of potential changes in axons and processes of neurons of a barnacle ganglion. J. Neurosci., in press.

Ross, W. N., B. M. Salzberg, I. B. Cohen, A. Grinvald, H. V. Davila, A. S. Waggoner, and C. H. Wang (1977) Changes in absorption, fluorescence, dichroism and birefringence in stained giant axons: Optical measurement of membrane potential. J. Membr. Biol. 33: 141-183.

Sakin, H., and E. L. Boulpaep (1981) Isolated perfused salamander proximal tubule: Methods, electrophysiology, and transport. Am. J. Physiol. 241: F39-F52.

Salzberg, B. M., A. Grinvald, L. B. Cohen, H. V. Davila, and W. N. Ross (1977) Optical recording of neuronal activity in an invertebrate central nervous system: Simultaneous monitoring of several neurons. J. Neurophysiol. 40: 1281-1291.

Shepherd, G. M. (1963) Neuronal systems controlling mitral cell excitability. J. Physiol. (Lond.) 168: 101-117.

Sokoloff, L. (1977) Relation between physiological function and energy metabolism in the central nervous system. J. Neurochem. 29: 13-26. 
Stewart, W. B., J. S. Kauer, and G. M. Shepherd (1979) Functional organization of rat olfactory bulb analyzed by the 2-deoxyglucose method. J. Comp. Neurol. 185: 715-734.

Waldow, U., M. C. Nowycky, and G. M. Shepherd (1981) Evoked potential and single unit responses to olfactory nerve volleys in the isolated turtle olfactory bulb. Brain Res. 211: 267-283.
Wong-Riley, M. (1979) Changes in the visual system of monocularly sutured or enucleated cats demonstrable with cytochrome oxidase histochemistry. Brain Res. 171: 11-28.

Wood, C. C. (1981) Application of dipole localization methods to source identification of human evoked potentials. Ann. N. Y. Acad. Sci. 388: 139-155. 